\title{
El níquel y su vínculo con el agrandamiento gingival: Revisión de la literatura
}

\section{Relationship of nickel and gingival enlargement: Review of the literature}

\author{
GÓIMEZ ARCILA V* \\ FANG MERCADO $\mathbf{L} * *$ \\ HERRERA HERRERA $A * * *$ \\ DÍAZ CABALLERO A****
}

\begin{abstract}
Gómez Arcila V, Fang Mercado L, Herrera Herrera A, Díaz Caballero A. El níquel y su vínculo con el agrandamiento gingival: Revisión de la literatura. Av Periodon Implantol. 2014; 26, 2: 83-89.
\end{abstract}

\begin{abstract}
RESUIMEN
El agrandamiento gingival es común entre los pacientes y puede ser causado por una variedad de factores etiológicos. La razón más común es la inflamación gingival provocada por mala higiene oral y alta carga bacteriana. Otros factores implicados son los medicamentos sistémicos, en donde se encuentran bloqueadores de los canales de calcio, inmunosupresores y anticonvulsivantes. Algunos crecimientos gingivales podrían estar asociados a condiciones hormonales como la pubertad, el embarazo o la diabetes o ser un síntoma de una enfermedad sistémica y en raras ocasiones el agrandamiento gingival se asocia a factores genéticos y hereditario llamado fibromatosis gingival. Otra causa del agrandamiento gingival se viene presentando en la consulta odontológica y es la relacionada con la aparatología ortodóncica fija, cuya causa específica no está totalmente definida aún. El presente artículo es una revisión narrativa cuyo objetivo es describir las generalidades del agrandamiento gingival y reportes en la literatura sobre la acción de la aparatología ortodóncica fija en el agrandamiento gingival, pues se presume del níquel como otro factor causal de esta patología.
\end{abstract}

PALABRAS CLAVE: Corrosión, biocompatibilidad, níquel, hiperplasia gingival (Decs Bireme).

\section{SUMMARY}

Gingival overgrowth is common among patients and can be caused by a variety of etiological factors. The most common reason is gingival inflammation caused by poor oral hygiene and high bacterial load. Other factors include systemic drugs, like channel blockers are calcium, immunosuppressants and anticonvulsants. Some gingival overgrowth are associated with hormonal conditions such as puberty, pregnancy or diabetes or be a symptom of a systemic disease and rarely the gingival overgrowth is associated with genetic factors, who is called hereditary gingival fibromatosis. Another cause of gingival overgrowth has been presented in dentistry and is related to fixed orthodontic appliances, whose specific cause is not fully defined yet. This article is a narrative review which aims to describe the generalities of gingival overgrowth and reports in the literature of the effect of fixed orthodontic appliances in gingival overgrowth because nickel probably is another factor causing this condition.

KEY WORDS: Corrosion, biocompatibility, nickel, gingival overgrowth (MesH).

Fecha de recepción: 26 de agosto de 2013.

Fecha de aceptación: 7 de octubre de 2013.

Grupo de Investigación GITOUC. Facultad de Odontología. Universidad de Cartagena. Campus de la Salud. Cartagena. Colombia. Odontóloga. Maestrante en Microbiología. Universidad de Cartagena.

Odontólogo, MSc Inmunología. Universidad de Cartagena.

*** Odontóloga, MSc Farmacología. Universidad de Cartagena.

**** Odontólogo. Universidad de Cartagena. Especialista en Periodoncia. Universidad Javeriana. Máster en Educación. Universidad del Norte. Doctor en Ciencias Biomédicas. Universidad de Cartagena. 


\section{INTRODUCCIÓN}

Las aleaciones metálicas con las que se elaboran los diversos aditamentos de la aparatología ortodóntica fija, al estar presentes en un medio acuoso como la cavidad oral, se exponen a interacciones iónicas que conllevan a la corrosión; este proceso posteriormente inducirá alteraciones de los tejidos orales (1-2). Actualmente, es poca la evidencia que reporta efectos adversos del tratamiento de ortodoncia; sin embargo, se ha descrito la aparición de descamación labial, eritema multiforme, estomatitis de contacto, gingivitis y agrandamiento gingival; siendo esta última alteración de alta frecuencia en la consulta odontológica (3).

El agrandamiento gingival (AG) inducido por ortodoncia, inicialmente fue descrito como una respuesta alérgica al níquel; metal ampliamente usado en el tratamiento ortodóntico (4). Sin embargo, recientes estudios reportan que los iones níquel presentes en los aditamentos ortodónticos posiblemente sean el factor etiológico de la proliferación fibroblástica característica del AG (5). Por tal motivo, la presente revisión tiene como objetivo describir el papel del níquel sobre el agrandamiento gingival en pacientes con aparatología ortodóncica fija.

\section{AGRANDAMIENTO GINGIVAL}

El AG se define como una lesión infiltrante, no neoplásica, que se caracteriza por un lento y progresivo incremento en el tamaño y volumen de las encías. Histológicamente, el agrandamiento gingival se caracteriza por la acumulación de tejido conectivo denso fibroso, esto se cree que es debido a un desequilibrio entre la síntesis y la degradación de matriz extracelular (compuesta principalmente de fibras colágeno) o debido a una alteración en la proliferación de los fibroblastos. Clínicamente, se presenta la encía de color rosa, firme, con diversos grados de apariencia (lisa, nodular, fibrosa); ésta, puede llegar a cubrir las coronas de los dientes por completo, y comúnmente se presenta en las zonas posteriores de los maxilares; aunque también se han reportado casos en las que aparece de forma generalizada. Esto conlleva a problemas estéticos, funcionales, dificultad en el habla, la oclusión y en la higiene bucal (6).

En cuanto a su origen, frecuentemente es el resultado de cambios inflamatorios inducidos por la acumulación prolongada de placa bacteriana. Sin embargo, los diferentes tipos de AG se deben a factores etiológicos específicos, ya sean locales o sistémicos. Existen casos de AG en mujeres gestantes debido a la carga hormonal (Figura 1), casos similares ocurren durante la pubertad o en individuos con diabetes (8). Algunos grupos de fármacos también inducen la aparición de AG; ejemplo de esto son los bloqueadores de los canales de calcio, inmunosupresores y anticonvulsivantes (Figura 2) (9). Sin embargo, existen situaciones donde el agente etiológico es desconocido, como la fibromatosis gingival, la cual es idiopática (7). En la Tabla 1, se describen los diversos tipos de AG y los agentes etiológicos que lo desencadenan.

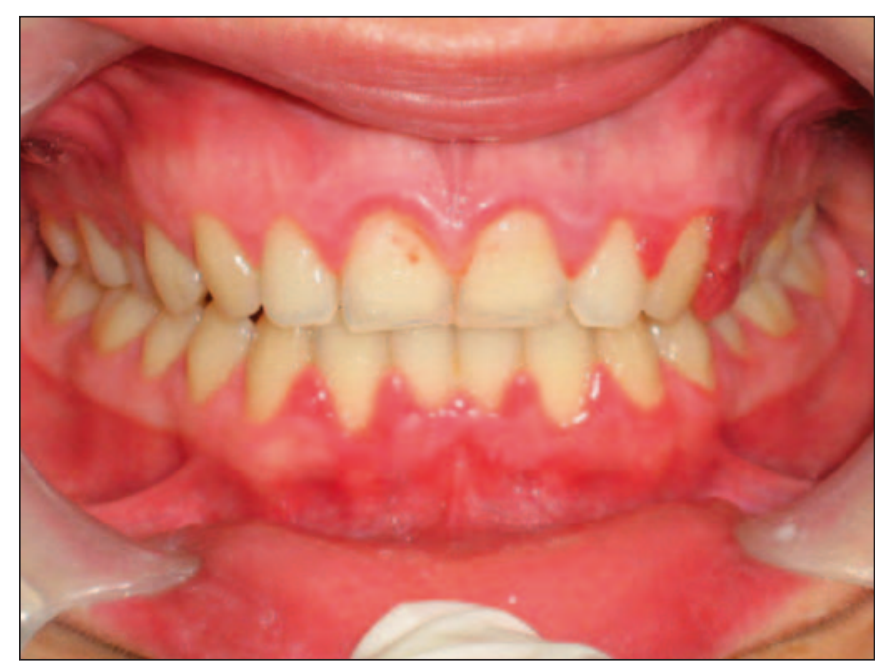

Fig. 1: Agrandamiento gingival durante el tratamiento de ortodoncia.

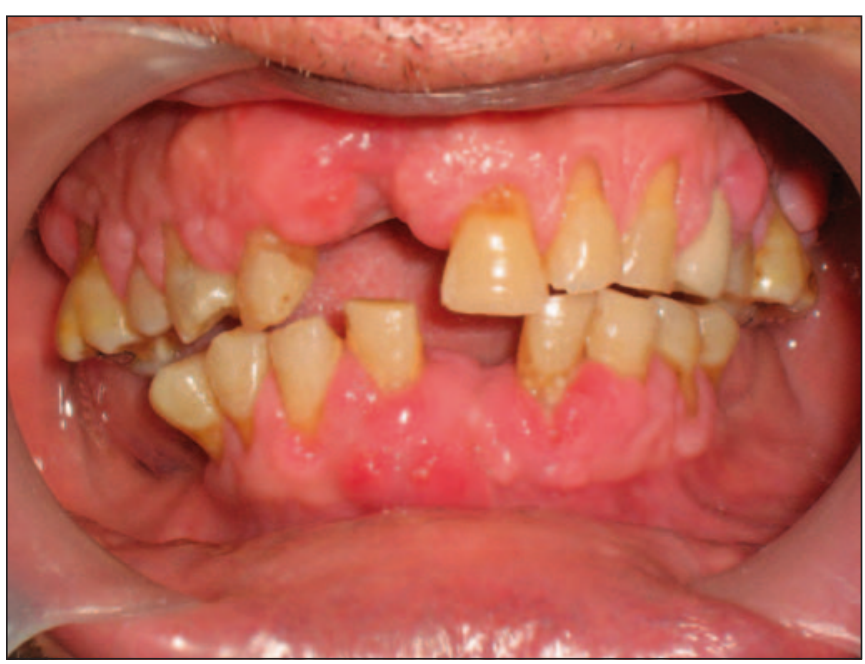

Fig. 2: Agrandamiento inducido por uso de fármacos anticonvulsivantes. 


\begin{tabular}{|l|l|}
\hline \multicolumn{2}{|l|}{ TABLA 1.- TIPOS DE AGRANDAMIENTO GINGIVAL Y SUS AGENTES ETIOLÓGICOS } \\
\hline Tipos de agrandamiento gingival & Agente etiológico \\
\hline A.G. Inflamatorio (12) & Exposición prolongada a placa bacteriana debido a mala higiene oral. \\
\hline A.G. inducido por fármacos (13) & $\begin{array}{l}\text { - Anticonvulsivantes: Ácido valproico y fenitoína. } \\
\text { - Inmunosupresores: Ciclosporina. } \\
\text { - Bloqueadores de los canales de calcio: Nifedipina, diltiacem, } \\
\text { felodipina, nitrendipina y verapamilo. }\end{array}$ \\
\hline A.G. condicionado (8, 14) & $\begin{array}{l}\text { - Hormonal: Embarazo, pubertad. } \\
\text { - Nutricional: Avitaminosis C. }\end{array}$ \\
\hline A.G. relacionado con enfermedades \\
sistémicas (15) & $\begin{array}{l}\text { Inespéfico: Granuloma piógeno. } \\
\text { - Leucemia. } \\
\text { Enfermedades granulomatosas: Granulomatosis deWegener, sarcoi- } \\
\text { dosis. }\end{array}$ \\
\hline A.G. neoplásico (16) & $\begin{array}{l}\text { Tumores benignos: Fibroma, papiloma, granuloma periférico de } \\
\text { células gigantes, leucoplasia, quiste gingival. } \\
\text { Tumores malignos: carcinoma, melanoma maligno. }\end{array}$ \\
\hline A.G. Falso (17) & $\begin{array}{l}\text { Lesiones óseas subyacentes: Torus, exostosis, enfermedad de Paget, } \\
\text { displasia fibrosa, querubismo, granuloma central de células gigan- } \\
\text { tes, osteoma, ameloblastoma. }\end{array}$ \\
\hline
\end{tabular}

Recientemente, se vienen reportando en la literatura, casos de AG en individuos con aparatología ortodóncica fija (Figura 3), generalmente durante las prime-

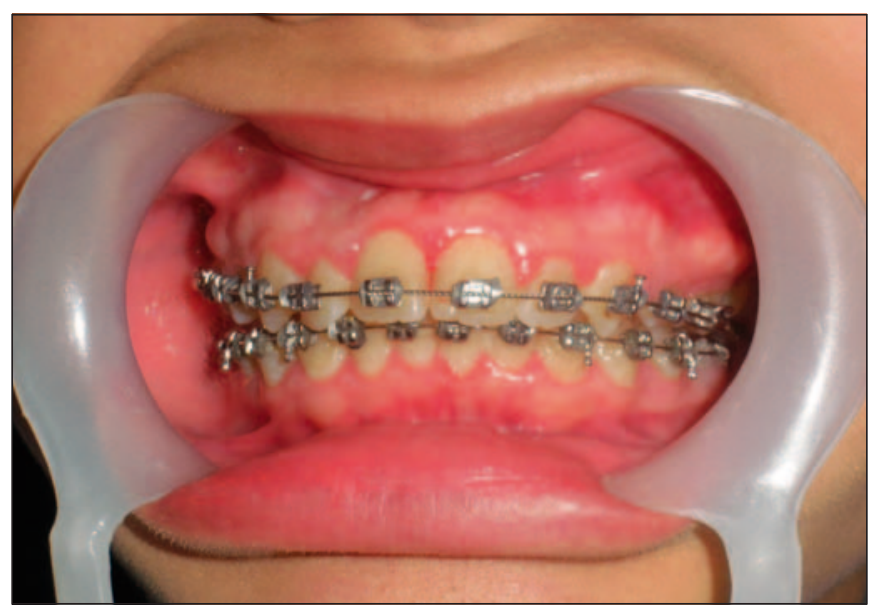

Fig. 3: Agrandamiento gingival inducido por ortodoncia. ras etapas del tratamiento; esta situación se explica por el acumulo de placa bacteriana y colonización de bacterias periodontopatógenas. Sin embargo, incluso con una higiene oral adecuada esta alteración persiste (10). Por lo que la causa precisa de este tipo de AG es tema de investigación actual.

Las aleaciones metálicas con las que se fabrican los aditamentos ortodónticos pueden estar induciendo la aparición de AG en pacientes bajo tratamiento ortodóntico. Ghon Y et al (2011) reportan que la irritación mecánica y química de las bandas y cementos empleados en el tratamiento ortodóntico, desencadenan el desarrollo de AG en este grupo de pacientes; además de la acumulación de detritos y una deficiente higiene oral. Estos factores desatan cambios en la composición de la microbiota oral que conllevan a una respuesta inflamatoria por parte del individuo (10). Contrario a esto, Johansson $\mathrm{K}$ et al (201 l) sugieren revisar la composición de las aleaciones metálicas y los porcentajes de los metales que para estos se emplean, más aún del níquel, pues es el metal más usado en la práctica 
odontológica y del cual se reporta bioacumulación en los tejidos orales (11).

\section{BIOCOIMPATIBILIDAD DEL NÍQUEL}

En odontología se emplean una amplia variedad de metales para la fabricación de prótesis dentales fijas y removibles, implantes de oseointegración, restauraciones directas e indirectas, así como también en la aparatología ortodóncica fija (18). Entre los metales comúnmente usados se encuentran: el oro, el cobalto, el cromo, el aluminio, el titanio, el hierro, el paladio, el platino, la plata, el osmio, el cobre, el cinc, el indio, el berilio, el estaño y el níquel (19).

Este último, presenta un aspecto blanco plateado, el cual es duradero, maleable y dúctil, con alta resistencia a la corrosión y oxidación (20). Gracias a la gran facilidad de permitir excelentes aleaciones con otros metales como el cromo y titanio, el níquel es de gran utilidad en la fabricación de la aparatología ortodóncica, en donde se combina con el acero inoxidable en un $8 \%$ (22). Sin embargo, los porcentajes de níquel presentes en los diferentes aditamentos ortodónticos como alambres, bandas y soportes dependen del tipo de aleación empleada por la casa fabricante (1). Un efecto adverso reportado, es la capacidad de sensibilización de la respuesta inmune del individuo, en comparación con los demás metales (21).

De otra parte, el concepto de biocompatibilidad de un metal, se relaciona con su capacidad de liberación iónica en un medio acuoso. Esta liberación se ve favorecida en ambientes cambiantes como la cavidad oral, que se caracteriza por fluctuaciones de temperatura, variaciones en las fuerzas de masticación, variedad de alimentos ingeridos, uso de pasta dental fluorada, saliva, presencia de microorganismos, entre otros; estas condiciones, al promover la biodegradación de los soportes, bandas, arcos y alambres de los aparatos de ortodoncia; conducen a efectos adversos a nivel local debido a que los iones de níquel son liberados, ocasionando desde reacciones alérgicas, genotoxicidad, citotoxicidad y por último bioacumulación $(3,23-24)$.

La alergia al níquel presente en los aparatos de ortodoncia fijos se conoce como "estomatitis por contacto" y constituye la reacción adversa local de mayor prevalencia (11). Sin embargo, la sensibilidad al níquel es menor en sujetos que han recibido tratamiento de ortodoncia, debido al desarrollo de tolerancia inmunológica durante todo el tratamiento (25). La respuesta alérgica a las aleaciones que contienen níquel es principalmente de tipo IV, reacción de hipersensibilidad mediada por células por los linfocitos T (1); se ha sugerido que la exposición a largo plazo a los materiales dentales con níquel puede afectar negativamente tanto monocitos humanos y células de la mucosa bucal (24).

Además de la alergia, alteraciones de citotoxicidad y genotoxicidad celular también se han reportado para el níquel. Algunos estudios han evaluado cambios en la respuesta a los aparatos de ortodoncia, encontrando daño del ADN en las células de la mucosa oral (2628). Natarajan et al (2011) determinaron el daño genotóxico de células de la mucosa bucal de pacientes con aparatología ortodóncica fija, concluyen que los iones níquel y cromo emitidos en cantidades suficientes inducen efectos genotóxicos localizados, pero estos cambios revierten cuando finaliza el tratamiento ortodóncico (29); hallazgos concordantes con los de Afees et al (2011) quienes concluyeron que los aparatos ortodóncicos fijos disminuyen la viabilidad celular, inducen daño en el ADN, y aumentan los contenidos de níquel y cromo en las células de la mucosa bucal (3). Estos reportes, sugieren que el daño causado al ADN no es permanente gracias a que se activan mecanismos para su reparación (30), y/o la eliminación de la fuente de genotoxicidad. Sin embargo, los mismos autores precisan que si el daño en el ADN persiste, dará lugar a inestabilidad genética y la célula se convierte en cancerosa debido a la acumulación de las mutaciones de la replicación del ADN dañado (31). Por tal motivo, se requiere una comprensión completa de la reparación del ADN, además, tratar a los pacientes con materiales ortodóncicos con menor potencial genotóxico, que contemplen normas más estrictas para la resistencia a la corrosión.

Diversos estudios han reportado el comportamiento de los metales liberados por los aparatos de ortodoncia bajo diversos cambios de condiciones físicas y químicas. Estos demuestran que los iones níquel liberados en muestras de saliva o de sangre son significativamente inferiores al promedio de ingesta dietaria y sin alcanzar concentraciones tóxicas (32-35). Sin embargo, concentraciones no tóxicas podrían ser suficientes para producir cambios biológicos en la mucosa oral, debido a los efectos citotóxicos que estos ocasionan $(3,24)$. Faccioni et al (2003) concluyo que los iones níquel rompen la cadena de ADN en células de la mucosa oral, alterando diversos mecanismos de protección y reparación que mantienen la homeostasis celular y la integridad del ADN; además de bioacumularse y ocasionar cambios irreversibles en las estructuras celulares (28). 


\section{BIOACUMULACIÓN DE NÍQUEL EN CAVIDAD ORAL}

La cantidad de níquel que se consume por vía oral como parte de la ingesta alimentaria, es importante, ya que el níquel es un oligoelemento esencial en la actividad enzimática, con relación a esto, algunos reportes señalan que los iones níquel liberados de las restauraciones metálicas y aparatos intraorales no se acumulan en el medioambiente oral (36), lo cual resulta discutible, pues recientes investigaciones han arrojado resultados concordantes con la bioacumulación de níquel en cavidad oral; una de las tantas reacción adversa local generada por la degradación de este metal.

Aunque estudios previos han evaluado la presencia de iones níquel en saliva y otros fluidos (37). Reportes recientes como el de Ousehal y Lazrak (2012), demostraron un aumento significativo de los niveles de níquel en saliva 8 semanas después de la inserción del arco de alambre de níquel-titanio (35). Estos hallazgos demuestran que la concentración de níquel en saliva es alterada por la aparatología ortodóncica fija.

Amini $\mathrm{F}$ et al (2008), mediante un estudio in vivo, evaluaron el contenido de $\mathrm{Ni}, \mathrm{Cr}$ y Co en células de mucosa oral de pacientes con y sin aparatos ortodóncicos fijos, empleando espectrofotometría de absorción atómica. Sus resultados reflejaron que el contenido de níquel en las muestras de mucosa fue significativamente mayor en los pacientes de ortodoncia en comparación con los controles (23). Estos resultados concuerdan con los de Faccioni et al (2003) quienes reportaron niveles relativamente altos de níquel en mucosa de pacientes sometidos a terapia ortodóncica, siendo de 3 a 4 veces mayor que en los sujetos sin terapia (28).

\section{MECANISMO DE AGRANDAMIENTO GINGIVAL INDUCIDO POR NÍQUEL}

Valeria de Paiva (2005) evaluó específicamente la relación del AG con hipersensibilidad al níquel en pacientes bajo tratamiento. Concluyó que, el examen clínico y periodontal no eran eficaces para el diagnóstico de alergia al níquel debido a que no se observó una asociación significativa entre la prueba del parche, el control de la placa bacteriana y las manifestaciones clínicas de AG (38). A pesar de ser el AG un indicador de reacción de hipersensibilidad al níquel, no es un signo específico de esta respuesta inmune, puesto que ocurre debido a otros eventos etiológicos: endocrinos, hormonales, medicamentos, enfermedades y sobre- carga bacteriana por inadecuado control de placa bacteriana (39). Por tanto, la posibilidad que exista AG ante la presencia de iones níquel acumulado en los tejidos y no por acumulación de placa bacteriana será necesario precisarlo con mas investigaciones.

Yu J et al (2012) evaluaron los mecanismos moleculares de la apoptosis de fibroblastos gingivales humanos, sus resultados demuestran que el níquel induce apoptosis de estas células a través de la activación de la caspase-3; además, genera citotoxicidad e inhibición de la proliferación celular dependiendo de la dosis (40). Por otro lado, Gursoy et al (2007) evaluaron la acumulación de níquel en los tejidos orales de pacientes con y sin agrandamiento gingival con una meticulosa higiene oral. Sus resultados no mostraron diferencia significativa en la acumulación del níquel en muestras con y sin agrandamiento gingival, pero afirman que bajo el análisis espectrofotométrico empleado, éste metal se bioacumula en tejido gingival, estimulando la proliferación de células epiteliales en respuesta a bajas concentraciones de níquel, mientras que a dosis más altas, se observó citotoxicidad (5). Estos estudios demuestran que el níquel si es capaz de inducir cambios morfológicos irreversibles.

La exposición prolongada y continua a los metales utilizados en odontología, pueden influir en la calidad de los tejidos y en los procesos bioquímicos que en estos ocurran. Por lo tanto, el interés por el níquel entre los investigadores no termina aún, pues este metal en cantidades excesivas puede conducir a efectos biológicos inesperados en cavidad oral alterando el equilibrio normal del organismo.

Aunque los aparatos de ortodoncia no parezcan tener una relación íntima con los tejidos orales, el ambiente particular de la cavidad oral potencia la corrosión y consecuente liberación de estos metales; siendo detectables en saliva, mucosa oral y encía; esta última revelada en pacientes ortodóncicos con presencia de AG. Aunque los estudios recientes no corroboren por completo esta asociación, es imprescindible dar a conocer los posibles efectos adversos locales que genera el níquel en cavidad oral y hacer hincapié en la conciencia que deben tener los pacientes sobre sus peligros potenciales.

\section{CONCLUSIÓN}

En conclusión, durante el tratamiento de ortodoncia, los iones níquel liberados podrían ser el factor de iniciación del agrandamiento gingival, pues está demos- 
trada la capacidad de aumentar la proliferación celular epitelial dependiendo del tiempo y la dosis. No obstante, esta patología en este grupo de pacientes, constituye un amplio campo de estudio que deberá precisarse con nuevas investigaciones sobre los mecanismos moleculares por el cual este metal se acumula en los tejidos orales, especialmente en el tejido gingival.

\section{AGRADECIMIENTOS}

Verónica Gómez, Alejandra Herrera, Luis Fang agradecen al Departamento Administrativo de Ciencia, Tecnología e Innovación -Colciencias- por su programa "Jóvenes investigadores".

\section{BIBLIOGRAFÍA}

1. Schmalz G, Garhammer P. Biological interactions of dental cast alloys with oral tissues. Dent Mater 2002;18:396406.

2. Hafez HS, Selim EM, Kamel Eid FH,TawfikWA, Al-Ashkar EA, Mostafa YA. Cytotoxicity, genotoxicity, and metal release in patients with fixed orthodontic appliances: a longitudinal in-vivo study. Am J Orthod Dentofacial Orthop 2011;140:298-308.

3. Ize-Iyamu IN, Ogbogu P. Nickel chromium brackets and its effect on the oral microflora. Afr J Med Med Sci 201 1; 40:367-71.

4. Mariotti A. Dental plaque-induced gingival diseases. Ann Periodontol 1999;4:7-19.

5. Gursoy UK, Sokucu O, Uitto VJ, Aydin A, Demirer S, Toker $\mathrm{H}$, et al. The role of nickel accumulation and epithelial cell proliferation in orthodontic treatment-induced gingival overgrowth. Eur J Orthod 2007;29:555-8.

6. Guiducci R, Vieira M, De Oliveira M, Chaves M, Lourenço A, Junior E. Tratamiento de la hiperplasia gingival en una escuela odontológica de Brasil. Conceptos generales, diagnóstico y tratamiento. Int J Odontostomat 2009;3:55-60.

7. Lai B, Muenzer J, Roberts MW. Idiopathic gingival hyperplasia: a case report with a 17-year followup. Case Rep Dent 2011;2011:986237.

8. McIntosh CL, Kolhatkar S, Winkler JR, Ojha J, Bhola M. An unusual case of generalized severe gingival enlargement during pregnancy. Gen Dent 2010;58: e272-8.
9. Livada R, Shiloah J. Gummy smile: could it be genetic? Hereditary gingival fibromatosis. J Tenn Dent Assoc 2012;92:23-6; quiz 27-8.

10. Gong $\mathrm{Y}, \mathrm{Lu} \mathrm{J}$, Ding X. Clinical, microbiologic, and immunologic factors of orthodontic treatment-induced gingival enlargement. Am J Orthod Dentofacial Orthop $2011 ; 140: 58-64$

11. Johansson K, Kerosuo H, Lammintausta K. Nickel sensitization in orthodontically treated and non-treated female adolescents. Contact Dermatitis 201 1;64:132-7.

12. Agrawal N, Agrawal K, Mhaske S. An uncommon presentation of an inflammatory gingival enlargementresponding to non-surgical periodontal therapy. Int J Dent Hyg 201 1;9:303-7.

13. Correa JD, Queiroz-Junior CM, Costa JE, Teixeira AL, Silva TA. Phenytoin-induced gingival overgrowth: a review of the molecular, immune, and inflammatory features. ISRN Dent 2011;2011:497850.

14. Shivaswamy S, Siddiqui N, Jain SA, Koshy A, Tambwekar S, Shankar A. A rare case of generalized pyogenic granuloma: a case report. Quintessence Int 201 1;42:493-9.

15. Bhatt V, Hall TJ. Strawberry gingival enlargement as only manifestation of Wegeners granulomatosis. Br J Oral Maxillofac Surg 2009;47:500.

16. Damm DD. Localized gingival enlargement. Adenomatoid odontogenic tumor. Gen Dent 2012;60(4): 355-7.

17. Kesic L, Mihailovic D, Pesic Z, Obradovic R. False gingival enlargement as a diagnostic problem: a case report. Int J Dent Hyg 2008;6:68-71.

18. Baumann B, Pai WH, Bennani V,Waddell JN. Dental alloys used for crown and bridge restorations by dental technicians in New Zealand. N Z Dent J 2010;106:43-9.

19. Raap U, Stiesch M, Kapp A. Contact allergy to dental materials.J Dtsch Dermatol Ges 2012;10:391-6; quiz 97.

20. Chaturvedi TP, Upadhayay SN. An overview of orthodontic material degradation in oral cavity. Indian J Dent Res 2010;21:275-84.

21. Kolokitha OE, Chatzistavrou E. Allergic reactions to nickel-containing orthodontic appliances: clinical signs and treatment alternatives. World J Orthod 2008;9:399406. 
22. Noble J, Ahing SI, Karaiskos NE, Wiltshire WA. Nickel allergy and orthodontics, a review and report of two cases. Br Dent J 2008;204:297-300.

23. Amini F, Borzabadi Farahani A, Jafari A, Rabbani M. In vivo study of metal content of oral mucosa cells in patients with and without fixed orthodontic appliances. Orthod Craniofac Res 2008;11:51-6.

24. Wataha JC, Lockwood PE, Schedle A, Noda M, Bouillaguet $\mathrm{S}$. Ag, $\mathrm{Cu}, \mathrm{Hg}$ and $\mathrm{Ni}$ ions alter the metabolism of human monocytes during extended low-dose exposures. J Oral Rehabil 2002;29:133-9.

25. Marigo M, Nouer DF, Genelhu MC, Malaquias LC, Pizziolo VR, Costa AS, et al. Evaluation of immunologic profile in patients with nickel sensitivity due to use of fixed orthodontic appliances. Am J Orthod Dentofacial Orthop 2003; 124:46-52.

26. Pereira BR, Tanaka OM, Lima AA, Guariza-Filho O, Maruo $\mathrm{H}$, Camargo ES. Metal and ceramic bracket effects on human buccal mucosa epithelial cells. Angle Orthod 2009;79:373-9.

27. Westphalen GH, Menezes LM, Pra D, Garcia GG, Schmitt VM, Henriques JA, et al. In vivo determination of genotoxicity induced by metals from orthodontic appliances using micronucleus and comet assays. Genet Mol Res 2008;7:1259-66.

28. Faccioni F, Franceschetti P, Cerpelloni M, Fracasso ME. In vivo study on metal release from fixed orthodontic appliances and DNA damage in oral mucosa cells. Am J Orthod Dentofacial Orthop 2003;124:687-93; discussion 93-4.

29. Natarajan M, Padmanabhan S, Chitharanjan A, Narasimhan M. Evaluation of the genotoxic effects of fixed appliances on oral mucosal cells and the relationship to nickel and chromium concentrations: an in-vivo study. Am J Orthod Dentofacial Orthop 201 1; 140: 383-8.

30. Beyersmann D, Hartwig A. Carcinogenic metal compounds: recent insight into molecular and cellular mechanisms. Arch Toxicol. 2008;82:493-512.

31. Ribeiro DA, Favero Salvadori DM, da Silva RN, Ribeiro Darros B, Alencar Marques ME. Genomic instability in non-neoplastic oral mucosa cells can predict risk during 4-nitroquinoline 1-oxide-induced rat tongue carcinogenesis. Oral Oncol 2004;40:910-5.
32. Gursoy S, Acar AG, Sesen C. Comparison of metal release from new and recycled bracket-archwire combinations. Angle Orthod. 2005;75:92-4.

33. Karnam SK, Reddy AN, Manjith CM. Comparison of metal ion release from different bracket archwire combinations: an in vitro study. J Contemp Dent Pract 2012;13:376-81.

34. Singh DP, Sehgal V, Pradhan KL, Chandna A, Gupta R. Estimation of nickel and chromium in saliva of patients with fixed orthodontic appliances. World J Orthod 2008;9:196-202.

35. Ousehal L, Lazrak L. Change in nickel levels in the saliva of patients with fixed orthodontic appliances. Int Orthod. 2012;10:190-7.

36. López-Alias JF, Martinez-Gomis J, Anglada JM, Peraire $\mathrm{M}$. Ion release from dental casting alloys as assessed by a continuous flow system: Nutritional and toxicological implications. Dent Mater 2006;22:832-7.

37. Kerosuo H, Moe G, Hensten-Pettersen A. Salivary nickel and chromium in subjects with different types of fixed orthodontic appliances. Am.J. Orthod. Dentofac. Orthop 1997 Jun; 111:595-8.

38. De Paiva VCX. Hipersensibilidade ao níquel presente no aparelho ortodôntico fixo metálico. Avaliação Clínica, Periodontal, Histopatológica E Imunoistoquímica. [Dissertação (Mestrado em Ortodontia]. Minas Gerais: Pontifícia Universidade Católica de Minas Gerais; 2005.

39. Benoist HM, Ngom PI, Seck-Diallo A, Diallo PD. [Gingival hypertrophy during orthodontic treatment: contribution of external bevel gingivectomy. Case report]. Odontostomatol Trop 2007;30:42-6.

40. Yu J, Zhao F, Wen X, Ding Q, Zhang L, Wang G. Apoptosis mechanism of gingival fibroblasts induced by nickel ion contained in dental cast alloys. Bio-medical materials and engineering 2012;22:151-7

\section{CORRESPONDENCIA}

\section{Antonio Díaz Caballero}

Facultad de Odontología Universidad de Cartagena. Campus de la Salud Zaragocilla.

Cartagena, Bolívar, Colombia

Correo electrónico: adiazcl@unicartagena.edu.co 